\title{
Associação entre ocorrência e fatores de risco de doenças crônicas não transmissíveis e insegurança alimentar pregressa em adultos do Distrito Federal
}

\section{Association between occurrence and risk factors of chronic noncommunicable diseases and past food insecurity in adults from the Federal District, Brazil}

Andressa Cristina S. de Deus'

Ana Lilian Santos²

Muriel Bauermann Gubert ${ }^{\top}$

1 Departamento de Nutrição, Faculdade de Ciências da Saúde. Universidade de Brasília. Brasilia-DF, Brasil.

2 Programa de Pós-Graduação em Saúde Coletiva, Faculdade de Ciências da Saúde. Universidade de Brasilia. Brasília-DF, Brasil.

Correspondência / Correspondence

Andressa Cristina Santos de Deus

E-mail: andressa.csd.nut@gmail.com

\section{Resumo}

Estudos apontam a possível relação entre insegurança alimentar (IA) e a ocorrência de doenças crônicas não transmissíveis (DCNTs) em adultos, além da relação entre privação de alimentos na infância e o desenvolvimento de obesidade na fase adulta. Porém, a associação entre IA vivida na infância e ocorrência de DCNTs em adultos ainda foi pouco estudada. O objetivo deste estudo foi verificar a associação entre IA pregressa (IAP) e a ocorrência de DCNT em adultos do Distrito Federal, Brasil. A amostra, representativa para a população do DF, incluiu 291 indivíduos acima de 18 anos. Para verificar essa associação, foi calculada a razão de prevalência das DCNTs entre indivíduos com segurança alimentar pregressa e IAP. Da amostra analisada, $56,4 \%$ apresentaram IAP, e destes, 52,7\% apresentaram excesso de peso/obesidade na vida adulta. Apesar de não significativos, a IAP aumentou o risco de ocorrência de doenças cardiovasculares em 14\% (RPA2 1,14; IC 95\%:0,65-2,00), diabetes em 57\% (RPA2 1,57; IC 95\%:0,64-3,88) e câncer em 52\% (RPA2 1,52; IC 95\%:0,376,29). Os dados mostram que tendência de que IAP esteja associada àocorrência de DCNTs em adultos. São necessários outros estudos para confirmar tal associação.

Palavras-chave: Segurança Alimentar. Infância. Doenças Crônicas. Obesidade. Prevalência.

\section{Abstract}

Studies indicate a possible link between food insecurity (FI) and the occurrence of chronic non-communicable diseases (NCDs) in adults, and also the relationship between food deprivation in 
childhood and development of obesity in adulthood. However, the association between FI experienced in childhood and the occurrence of NCDs in adults has been little studied. This study aimed to investigate the association between past FI (PFI) and the occurrence of NCDs in adults from the Federal Disctrict (FD), Brazil. The sample is representative for the population of FD and included 291 individuals over 18 yearsold. To verify this association, the prevalence ratio of NCDs among individuals with previous food security and PFI was calculated. Of the analyzed sample, $56.4 \%$ presented IAP, and of these, $52.7 \%$ had overweight / obesity in adulthood. Although not significant, the PFI increased risk of cardiovascular disease by 14\% (APR2 1.14, 95\% CI :0,65-2, 00 ), diabetes by $57 \%$ (APR2 1.57, 95\% CI :0,64-3, 88) and cancer by 52\% (APR2 1.52, 95\% CI :0,37-6, 29). Data show a tendency of PFI being associated with the occurrence of NCDs in adults. Further studies are needed to confirm this association.

Key words: Food Security. Childhood. Chronic Diseases. Obesity. Prevalence.

\section{Introdução}

A segurança alimentar caracteriza-se como sendo o direito humano ao acesso regular e contínuo a alimentos saudáveis e de qualidade, em quantidades suficientes, levando-se também em consideração aspectos culturais e sustentabilidade ambiental e socioeconômica. ${ }^{1}$ Engloba processos desde a produção agrícola até o acesso da população aos alimentos, todos relacionados ao conceito de segurança alimentar. ${ }^{2}$

A insegurança alimentar (IA), portanto, condiz com situações em que fatores acabam por impedir que o indivíduo tenha acesso a alimentos adequados, promovendo situações de carências alimentares e, em estágios mais avançados, fome. ${ }^{3} \mathrm{O}$ grau de IA da população pode ser aferido por diversos indicadores, dentre eles a Escala Brasileira de Insegurança Alimentar (EBIA), constituída de 14 perguntas, que gera uma classificação de quatro categorias de condição de segurança alimentar domiciliar. ${ }^{4}$ De acordo com a Pesquisa Nacional por Amostras de Domicílio (PNAD) de 2009, 30,2\% dos domicílios brasileiros estavam em condição de IA, compreendendo cerca de 65,6 milhões de pessoas. Do total de domicílios avaliados, 18,7\% apresentavam IA leve, $6,5 \%$ IA moderada e $5 \%$ IA grave. ${ }^{4}$ 
Alguns estudos mostram a associação existente entre situações de IA e sobrepeso e obesidade, ${ }^{5-8}$ e outras doenças crônicas não transmissíveis (DCNTs), como diabetes e hipertensão. ${ }^{9-12}$ Confirmam, assim, que carências nutricionais e fome não são as únicas formas de expressão desta condição. ${ }^{3}$ Além disso, estudos apontam a relação entre situações de privação de acesso a alimentos, ocorridas no passado do indivíduo, como desencadeadoras da presença de obesidade em adultos. ${ }^{13-18}$ No entanto, a possível relação existente entre a experiência de insegurança alimentar durante a infância e a ocorrência de DCNTs na vida adulta ainda não foi muito estudada.

Este trabalho tem como objetivo avaliar a associação entre a existência de insegurança alimentar pregressa (IAP) na infância e a ocorrência de DCNT em indivíduos adultos, do Distrito FederalDF, Brasil.

\section{Metodologia}

Foi realizado estudo transversal no primeiro semestre de 2014, com população adulta no Distrito Federal (DF). A amostra foi aleatória e estratificada por situação de insegurança alimentar e faixa de renda familiar domiciliar. ${ }^{4}$ Durante a etapa de desenho amostral, foram realizados os procedimentos de classificação da população, determinação da proporção para cada classe de renda e fixação das cotas amostrais em observância à proporção das classes consideradas. O tamanho amostral calculado foi de 291 indivíduos (homens ou mulheres), com erro máximo de 10\% e nível de confiança de 95\%. A amostra estudada possui representatividade para indivíduos com segurança alimentar e insegurança alimentar grave no DF.

Dentre os critérios de exclusão estavam: gravidez, ser surdo ou mudo ou ser portador de necessidades especiais. Já os critérios de inclusão foram: ter idade igual ou superior a 18 anos, residir no DF e ter conhecimento sobre a disponibilidade de alimentos no domicílio, ou seja, envolvimento com a compra, armazenamento ou consumo regular dos alimentos no domicílio. Os dados foram coletados em entrevistas face a face, em locais de grande circulação populacional do DF.

O instrumento utilizado foi validado em estudo piloto e era composto por: 1) questionário de triagem, quando os indivíduos eram abordados sistematicamente e convidados a participar da pesquisa, sendo coletada renda familiar mensal e aplicado o questionário da Escala Brasileira de Insegurança Alimentar (EBIA), ${ }^{19,20}$ com o objetivo de avaliar o grau de segurança alimentar dos indivíduos e, assim, selecionar os indivíduos que cumpriam os critérios de inclusão; e 2) questionário composto por perguntas fechadas, oriundas do questionário utilizado na pesquisa VIGITEL 2011, para avaliação dos fatores de risco e proteção envolvidos na ocorrência de DCNTs, ${ }^{16}$ e a EBIA pregressa, composta por sete perguntas referentes à experiência de insegurança alimentar durante a infância, aos 12 anos de idade, conforme empregado em estudo anterior. ${ }^{17}$ 
O questionário da EBIA aplicado na etapa de triagem era composto por 14 perguntas referentes a vivência e/ou percepção de insuficiência alimentar e fome em nível domiciliar em relação aos últimos três meses que precederam a entrevista. ${ }^{4}$ As perguntas do questionário pós-triagem abordavam: a) características demográficas e socioeconômicas dos indivíduos; b) padrão de alimentação e de atividade física; c) peso e altura referidos; d) perguntas relacionadas à situação de saúde na infância; e) consumo de bebidas alcoólicas; f) autoavaliação do estado de saúde e diagnóstico médico anterior de DCNT; g) questionário de SAP para captar a possível influência das condições de segurança alimentar em relação ao acesso aos alimentos durante a infância do indivíduo.

Para análise do status de segurança alimentar dos indivíduos na infância, aqueles que responderam "não" a todas as sete questões da EBIA pregressa foram classificados com segurança alimentar pregressa (SAP). Já os indivíduos que responderam "sim" a uma ou mais questões foram classificados com insegurança alimentar pregressa (IAP).

O presente estudo foi aprovado pelo Comitê de Ética em Pesquisa da Universidade de Brasília, parecer número 392.831. Os indivíduos que aceitaram participar da pesquisa assinaram o Termo de Consentimento Livre e Esclarecido.

As estimativas fornecidas pelas análises bivariadas (relação entre insegurança alimentar pregressa e variáveis socioeconômicas, e presença de doenças crônicas) foram expressas em razões de prevalência (RP) e intervalos de confiança de 95\%, sendo aplicado o teste de qui-quadrado para a comparação das propoções de IAP observadas. Para análise ajustada, foi utilizada a regressão de Poisson. As variáveis com $\mathrm{p}<0,20$ e situação de segurança alimentar atual foram inseridas como variáveis de controle. O nível de significância considerado foi de $5 \%$.

\section{Resultados}

Neste estudo, foram entrevistados 291 indivíduos adultos residentes no Distrito Federal. Em relação aos dados de idade e raça, ocorreu a perda dos dados de dois participantes que se recusaram a fornecer as informações sobre as variáveis citadas, conforme destacado na tabela 1.

A tabela 1 mostra que 56,4\% apresentaram insegurança alimentar pregressa. A maior parte da amostra pertencia ao sexo feminino $(52,9 \%)$ e a média de idade dos respondentes foi de 38 anos (desvio padrão $\pm 15,86$ anos). Em relação ao nível de escolaridade dos participantes do estudo, 48,5\% cursaram até o ensino médio. Comparando-se os dois grupos (SAP e IAP), observou-se que o grupo de indivíduos que tiveram IAP apresentou maior prevalência de excesso de peso/obesidade na vida adulta (52,7\%). Considerando-se a amostra total, o valor de IMC médio obtido para homens e mulheres foi, respectivamente, 24,98 e $25,37 \mathrm{~kg} / \mathrm{m}^{2}$, ambos classificados como sobrepeso. 
Tabela 1. Características sociodemográficas da amostra segundo situação de segurança alimentar pregressa. Brasília-DF, 2014.

\begin{tabular}{|c|c|c|c|c|c|c|c|}
\hline \multirow[b]{3}{*}{ Sexo } & \multirow[b]{2}{*}{$\mathrm{n}$} & \multirow[b]{2}{*}{$\%$} & \multicolumn{2}{|c|}{$\begin{array}{l}\text { Segurança } \\
\text { Alimentar } \\
\text { Pregressa }\end{array}$} & \multicolumn{2}{|c|}{$\begin{array}{c}\text { Insegurança } \\
\text { Alimentar } \\
\text { Pregressa }\end{array}$} & \multirow[t]{2}{*}{$\mathrm{p}$-valor ${ }^{\mathrm{e}}$} \\
\hline & & & $\mathrm{n}$ & $\%$ & $\mathrm{n}$ & $\%$ & \\
\hline & & & & & & & \\
\hline Feminino & $(154)$ & 52,9 & (64) & 50,4 & $(90)$ & 54,9 & \\
\hline Masculino & $(137)$ & 47,1 & (63) & 49,6 & (74) & 45,1 & 0,11 \\
\hline Idade* & & & & & & & 0,021 \\
\hline $18-29$ & $(105)$ & 36,1 & $(59)$ & 46,8 & $(46)$ & 28,2 & \\
\hline $30-39$ & (53) & 18,3 & (20) & 15,9 & (33) & 20,2 & \\
\hline $40-49$ & (62) & 21,5 & (23) & 18,3 & (39) & 23,9 & \\
\hline $50-59$ & (34) & 11,8 & $(10)$ & 7,9 & (24) & 14,7 & \\
\hline 60 ou mais & (35) & 12,1 & (14) & 11,1 & (21) & 12,9 & \\
\hline Média de Idade (DP) & (DP & $\begin{array}{l}\operatorname{nos} \\
\left.86^{* * * *}\right)\end{array}$ & $\begin{array}{r}35 \\
(\mathrm{DP}=\end{array}$ & & $\begin{array}{c}41 \text { ar } \\
(\mathrm{DP} \pm 1\end{array}$ & & \\
\hline Escolaridade & & & & & & & 0,342 \\
\hline Até ensino fundamental & (73) & 25,1 & $(21,3)$ & 27 & $(46)$ & 28,0 & \\
\hline Até ensino médio & $(141)$ & 48,5 & $(47,2)$ & 60 & $(81)$ & 49,4 & \\
\hline Até ensino superior & (63) & 21,6 & $(26,8)$ & 34 & (29) & 17,7 & \\
\hline Pós graduação & (8) & 2,7 & $(3,1)$ & 4 & (4) & 2,4 & \\
\hline Não estudou & (6) & 2,1 & $(1,6)$ & 2 & (4) & 2,4 & \\
\hline Raça**, & & & & & & & 0,947 \\
\hline Branco & (71) & 24,4 & (32) & 25,4 & (39) & 23,9 & \\
\hline Negro/Pardo & (207) & 71,1 & (89) & 70,6 & (118) & 72,4 & \\
\hline Amarelo/Indígena & (11) & 3,8 & (5) & 4,6 & (6) & 3,7 & \\
\hline
\end{tabular}




\begin{tabular}{|c|c|c|c|c|c|c|c|}
\hline Atualmente empregados, & $(229)$ & 78,7 & $(98)$ & 77,2 & $(131)$ & 79,9 & 0,575 \\
\hline \multicolumn{8}{|l|}{ Renda domiciliar per capita, } \\
\hline Até 1/4 salário mínimo & (20) & 6,9 & (3) & 2,4 & (17) & 10,4 & \multirow[t]{6}{*}{0,004} \\
\hline Mais de 1/4, até $1 / 2$ salário mínimo & $(49)$ & 16,8 & $(15)$ & 11,8 & (34) & 20,7 & \\
\hline Mais de 1/2, até 1 salário mínimo & $(72)$ & 24,7 & $(31)$ & 24,4 & $(41)$ & 25,0 & \\
\hline Mais de 1 , até 2 salários mínimos & $(79)$ & 27,1 & $(36)$ & 28,3 & $(43)$ & 26,2 & \\
\hline Mais de 2, salários mínimos & $(67)$ & 23 & (39) & 30,7 & (28) & 17,1 & \\
\hline Sem rendimento & (4) & 1,4 & (3) & 2,4 & (1) & 0,6 & \\
\hline \multicolumn{8}{|l|}{ Estado Civil, } \\
\hline Solteiro/Separado/Viúvo & $(176)$ & 60,5 & $(90)$ & 70,9 & (86) & 52,4 & \multirow[t]{2}{*}{0,001} \\
\hline Casado/União estável & $(115)$ & 39,5 & $(37)$ & 29,1 & (78) & 47,6 & \\
\hline IMC Homens, média, $\mathrm{kg} / \mathrm{m}^{2 * * *}$ & \multicolumn{2}{|c|}{24,98} & \multicolumn{2}{|c|}{24,25} & \multicolumn{2}{|c|}{25,72} & \\
\hline IMC Mulheres, média, $\mathrm{kg} / \mathrm{m}^{2 * * *}$ & \multicolumn{2}{|c|}{25,37} & \multicolumn{2}{|c|}{24,99} & \multicolumn{2}{|c|}{25,76} & \\
\hline Excesso de peso/obesidade*** & $(140)$ & 49,1 & $(55)$ & 44,4 & (85) & 52,8 & 0,061 \\
\hline \multicolumn{8}{|l|}{ Classificação de Segurança } \\
\hline Alimentar Pregressa $(n=291)$ & \multicolumn{2}{|c|}{$100 \%$} & \multicolumn{2}{|c|}{$43,6 \%$} & \multicolumn{2}{|c|}{$56,4 \%$} & \\
\hline
\end{tabular}

Amostra total $(\mathrm{n}=291) ; *$ Idade $(\mathrm{n}=289) ;$ **Raça $(\mathrm{n}=289) ; * * *$ IMC/Excesso de peso/obesidade $(\mathrm{n}=285) ; * * * *$ Desvio Padrão\&calculado pelo teste de Qui-Quadrado (comparação entre Segurança e Insegurança Alimentar pregressa)

A tabela 2 apresenta a prevalência de DCNTs segundo situação de SAP. Entre os indivíduos com SAP, a prevalência de doenças cardiovasculares foi de 16,5\%, de diabetes mellitus tipo 2 foi de $5,5 \%$ e de câncer foi de 2,4\%. No grupo de IAP, a prevalência destas doenças foi sempre maior. Entretanto, os resultados não foram estatisticamente significativos, mesmo quando ajustados para idade, renda per capita, estado civil, IMC e classificação atual de segurança alimentar. 
Tabela 2. Prevalência e razão de prevalência da associação entre segurança alimentar pregressa e doenças crônicas entre moradores do Distrito Federal. Brasília-DF, 2014.

\begin{tabular}{|c|c|c|c|c|c|}
\hline & $\begin{array}{c}\text { Segurança } \\
\text { Alimentar } \\
\text { Pregressa } \\
\text { Prevalência } \\
\text { não ajustada, } \\
\%\end{array}$ & $\begin{array}{c}\text { Insegurança } \\
\text { Alimentar } \\
\text { Pregressa } \\
\text { Prevalência não } \\
\text { ajustada, \%Razão } \\
\text { Prevalência Bruta } \\
\text { (IC 95\%)* }\end{array}$ & $\begin{array}{c}\text { Razão } \\
\text { Prevalência } \\
\text { Bruta (IC 95\%)* }\end{array}$ & $\begin{array}{c}\text { Razão } \\
\text { Prevalência } \\
\text { Ajustada 1 (IC } \\
\text { 95\%)** }\end{array}$ & $\begin{array}{c}\text { Razão } \\
\text { Prevalência } \\
\text { Ajustada } 2 \text { (IC } \\
95 \% \text { )*** }\end{array}$ \\
\hline $\begin{array}{c}\text { Doenças } \\
\text { Cardiovasculares } \\
\mathrm{n}=291\end{array}$ & 16,5 & 24,4 & $1,48(0,92-2,37)$ & $1,25(0,72-2,18)$ & $1,14(0,65-2,00)$ \\
\hline $\begin{array}{l}\text { Diabetes } \\
\mathrm{n}=291\end{array}$ & 5,5 & 10,4 & $1,88(0,81-4,4)$ & $1,61(0,66-3,94)$ & $1,57(0,64-3,88)$ \\
\hline $\begin{array}{l}\text { Câncer } \\
\text { n = } 291\end{array}$ & 2,4 & 4,3 & $1,81(0,48-6,85)$ & $1,71(0,43-6,84)$ & $1,52(0,37-6,29)$ \\
\hline
\end{tabular}

*Risco relativo para adultos com insegurança alimentar pregressa comparado com adultos com segurança alimentar pregressa. **Razão de prevalência ajustada 1 éajustada para idade, renda per capita, estado civil e IMC. ***Razão de prevalência 2 éajustada para idade, renda per capita, estado civil e IMC e classificação atual de segurança alimentar.

\section{Discussão}

A insegurança alimentar pregressa corresponde a situações relacionadas à alimentação vividas durante a infância. Cabe, portanto, considerar neste trabalho o viés de memória, apesar de se utilizar um questionário validado anteriormente. ${ }^{17}$

Sabe-se que situações vividas pelos indivíduos durante a infância podem influenciar a ocorrência de DCNTs durante a vida adulta, como, por exemplo, o imprinting metabólico, fenômeno no qual experiências nutricionais precoces em períodos críticos do desenvolvimento do indivíduo - gestação e infância - podem gerar efeitos futuros que o predisponham ao desenvolvimento de certas doenças. ${ }^{18} \mathrm{O}$ excesso de peso / obesidade, doenças cardiovasculares, dentre outras, na vida adulta, são exemplos desses desfechos. 
Autores têm apontado que situações de privação, tanto quantitativa quanto qualitativa, ao acesso a alimentos durante a infância estão associadas ao desencadeamento da obesidade em adultos. $^{13-15}$ Sendo a obesidade um dos fatores de risco para desenvolvimento de DCNTs, pode-se levantar a hipotese de que o risco para o desenvolvimento dessas doenças em indivíduos expostos a situações de IAP pode ser também aumentado, conforme a tendência dos resultados observados neste estudo, apesar de não significativos.

Observa-se que existe a tendência de que a experiência de insegurança alimentar na infância do indivíduo aumente o risco para o desenvolvimento dessas DCNTs durante a fase adulta. Alguns estudos já mostraram a existência de associação entre insegurança alimentar e ocorrência de algumas doenças crônicas não transmissíveis na fase adulta, como diabetes e hipertensão. ${ }^{9-12}$ No entanto, esses estudos consideraram o status de insegurança alimentar atual, e não pregressa, dos indivíduos estudados. Em virtude dos dados encontrados, sugere-se que são necessários outros estudos voltados para a insegurança alimentar pregressa, a fim de que se confirme a hipótese apontada por este trabalho.

\section{Referências}

1. Brasil. Lei n. ${ }^{\circ} 11.346$, de 15 de setembro de 2006. Cria o Sistema Nacional de Segurança Alimentar e Nutricional com vistas em assegurar o direito humano àalimentação adequada e dáoutras providências. Diário Oficial da União 18 set. 2006.

2. Gubert MB, Benicio MHDA, Santos LMP. Estimativas de insegurança alimentar grave nos municípios Brasileiros. Cad Saúde Pública. 2010; 26(8):1595-1605.

3. Kepple AW, Segall-Corrêa AM. Conceituando e medindo segurança alimentar e nutricional. Ciên. Saúde Coletiva. 2011; 16(1):187-199.

4. Fundação Instituto Brasileiro de Geografia e Estatística. Pesquisa Nacional por Amostra de Domicílios 2004-2009: Segurança alimentar. Rio de Janeiro: IBGE; 2010.

5. Olson CM. Nutrition and health outcomes associated with food insecurity and hunger. J. Nutr. 1999; 129(Suppl.2): 5215-45.

6. Alaimo K, Olson CM, Frongillo EA. Food insufficiency and American school-aged children's cognitive, academic, and psychosocial development. Pediatrics 2001; 108:144-53.

7. Townsend M, Peerson J, Love B, Achterberg C, Murphy S. Food insecurity is positively related to overweight in women. J. Nutr. 2001; 131(6):1738-1745.

8. Schlussel MM, Silva AAM, Pérez-Escamilla R, Kac G. Household food insecurity and excess weight/ obesity among Brazilian women and children: a life-course approach.Cad. Saúde Pública 2013; 29(2):219-26. 
9. Holben DH, Pheley AM. Diabetes risk and obesity in food-insecure households in rural Appalachian Ohio. Prev. Chronic Dis. 2006; 3(3):A82.

10. Seligman HK, Bindman AB, Vittinghoff E, Kanaya AM, Kushel MB. Food insecurity is associated with diabetes mellitus: results from the National Health Examination and Nutrition Examination Survey 1999-2002. J. Gen. Intern. Med. 2007; 22:1018-23.

11. Seligman HK, Bindman AB, Vittinghoff E, Kanaya AM, Kushel MB. Food insecurity Is associated with Chronic Disease among low-income NHANES participants. J. Nutr. 2010; 140:304-310.

12. Terrell CA, Drew C, Vargas R. Is food insecurity associated with chronic disease and chronic disease control? Ethn. Dis. 2009; 19: S3-6.

13. Sarlio-Lahteenkorva S, Lahelma E. Food insecurity is associated with past and present economic disadvantage and body mass index. J Nutr. 2001; 131:2880-2884.

14. Okasha M, Mccarron P, Mcewen J, Durnin J, Davey S. Childhood social class and adulthood obesity: findings from the Glasgow alumni cohort. J. Epidemiol. Community Health. 2003; 57(7):508-509.

15. Kaiser Ll, Townsend Ms, Melgar-Quiñoez Hr, Fuji Ml, Crawford Pc. Choice of instrument influences relationship between food insecurity and obesity in Latino women. Am. J. Clin. Nutr. 2004; 80:13721378 .

16. Brasil. Ministério da Saúde. Vigitel Brasil 2011: Vigilância de fatores de risco e proteção para doenças crônicas por inquérito telefônico. Brasília: Ministério da Saúde; 2012.

17. Souza-Esquerdo VF, Bergamasco SMPP, Oliveira JTA, Oliveira ES. Segurança alimentar e nutricional e qualidade de vida em assentamentos rurais. Segurança Alimentar e Nutricional. 2013; 20(1):13-23.

18. Waterland Ra, Garza C. Potencial mechanisms of metabolic imprinting that lead to chronic disease. Am. J. Clin. Nutr.1999; 69(2):179-97.

19. Pérez-Escamilla R, Segall-Corrêa AM, Maranha LK, Sampaio MFA, Marín L, Panigassi G. An adapted version of the US Departament of Agriculture Food insecure module is a valid tool for assessing food insecurity in Campinas, Brasil. J. Nutr. 2004;134:1923-1928.

20. Segall-Corrêa AM, Marin-Leon L. A Segurança alimentar no Brasil: proposição e usos da escala brasileira de medida da insegurança alimentar (EBIA) de 2003 a 2009. Segurança Alimentar e Nutricional 2009; 16(2):1-19. 
